\title{
Comparación entre la Componente Atmosférica del Sistema HWRF y el Modelo WRF-HWRF Utilizando Diferentes Resoluciones Horizontales en la Simulación del Huracán Irma (2017). Parte I
}

\author{
Albenis Pérez-Alarcón ${ }^{1,4}$ (D), Oscar Díaz-Rodríguez ${ }^{2}$ (D), José Carlos Fernández-Alvarez ${ }^{1,4}$ (D), \\ Ramón Pérez-Suárez ${ }^{3}$ D, Patricia Coll-Hidalgo ${ }^{1}$ \\ ${ }^{1}$ Departamento de Meteorología, Instituto Superior de Tecnologías y Ciencias Aplicadas, \\ Universidad de La Habana, La Habana, Cuba. \\ ${ }^{2}$ Centro de Ciencias de la Atmósfera, Universidad Autónoma de México, \\ Ciudad de México, México. \\ ${ }^{3}$ Instituto de Meteorología, La Habana, La Habana, Cuba. \\ ${ }^{4}$ Environmental Physics Laboratory, Centro de Investigación Marina, Universidad de Vigo, \\ Ourense, España.
}

Recibido em: 28 de Noviembre de 2019 - Aceptado em: 3 de Octubre de 2020

\begin{abstract}
Resumen
Se realizó un estudio de caso de varias configuraciones de modelos de pronóstico numérico para evaluar la habilidad de los mismos en el pronóstico de la intensidad y trayectoria de los ciclones tropicales. Para ello se seleccionaron 4 configuraciones del dominio de cómputo con 27-9 y 18-6 km de resolución para el HWRF (Hurricane Weather Research and Forecasting Model) y 4 configuraciones para el WRF (Weather Research and Forecasting Model), empleando el núcleo dinámico NMM (Non-hydrostatic Mesoscale Model) con la opción de seguimiento de vórtice. Se realizaron las simulaciones correspondientes al huracán Irma desde el 1ro al 12 de septiembre del 2017 inicializadas con salidas de pronóstico del GFS (Global Forecast System). En la evaluación realizada no se observaron diferencias notables entre las 8 configuraciones, aunque fue deficiente el pronóstico de la intensidad del huracán Irma, con un error en el pronóstico de la velocidad máxima del viento superior a los $50 \mathrm{~km} / \mathrm{h}$. La comparación de las salidas de cada configuración con los registros de las boyas y estaciones meteorológicas de superficie evidenció que el comportamiento de las variables viento y presión atmosférica tiene una tendencia similar a los valores registrados en las estaciones, con errores inferiores a los $3.8 \mathrm{~m} / \mathrm{s}$ para la velocidad del viento y $3 \mathrm{hPa}$ para la presión atmosférica. La configuración que mostró mejor habilidad para el pronóstico de ciclones tropicales fue HWRF_18-6-m (referida al modelo y la resolución horizontal empleada), aunque es la que más capacidad de cómputo requiere.
\end{abstract}

Palabras-clave: NMM, HWRF, ciclones tropicales, trayectoria, intensidad, pronóstico de huracanes.

\section{A Comparison Between the Atmospheric Component of HWRF System and WRF-HWRF Model Using Different Horizontal Resolutions in Hurricane Irma (2017) Simulation. Part I}

\begin{abstract}
A study of several configurations of numerical forecast models was conducted to evaluate their skill to forecast tropical cyclones track and intensity. For this, four configurations of the horizontal domain were selected with 27-9 and 18-6 km of resolution for HWRF (Hurricane Weather Research and Forecasting Model) and four configurations for the WRF (Weather Research and Forecasting Model), using the dynamic core NMM (Non-hydrostatic Mesoscale Model) with the vortex tracker option. Simulations corresponding to Hurricane Irma were done from $1^{\text {st }}$ to $12^{\text {th }}$ September 2017, initi-
\end{abstract}

Autor por correspondencia: Albenis Pérez-Alarcón, albenisp@instec.cu. 
alized with GFS (Global Forecast System) forecast outputs. No notable differences were observed between the eight configurations, although the forecast of the intensity of Hurricane Irma was deficient, with an error in the forecast of the maximum wind speed above $50 \mathrm{~km} / \mathrm{h}$. The comparison of the outputs of each configuration with buoys and surface meteorological stations data showed that the behavior of the wind speed and atmospheric pressure variables has a similar tendency to the registered values in the stations, with errors lower than $3.8 \mathrm{~m} / \mathrm{s}$ for wind speed and $3 \mathrm{hPa}$ for atmospheric pressure. HWRF 18-6-m (referred to the model and the horizontal domain resolution) was the configuration that showed the best ability for tropical cyclones forecast, although it require large time to compute.

Keywords: NMM, HWRF, tropical cyclones, track, intensity, hurricanes forecast.

\section{Introducción}

Los ciclones tropicales son conocidos como uno de los sistemas naturales más devastadores que existen. En muchos países conocen de sus efectos y de las grandes pérdidas de vidas humanas y materiales que ocasionan. Uno de sus principales impactos se produce en áreas costeras como consecuencia de los fuertes vientos superficiales, los tornados asociados y las inundaciones ocasionadas por las intensas lluvias y la marea de tormenta (Lighthill, 1998).

El pronóstico de intensidad y trayectoria de los mismos es un imperativo para la comunidad científica internacional con el objetivo de minimizar sus efectos. En la cuenca del Atlántico Norte, el centro regional para el pronóstico de los ciclones tropicales es el NHC (U. S. National Hurricane Center), que utiliza varias herramientas, entre ellas, la información de varios modelos operacionales de predicción de huracanes para confeccionar sus pronósticos oficiales. Los insuficientes avances en la habilidad de los modelos en el pronóstico de intensidad de ciclones tropicales condujeron a que la NOAA (U.S. National Oceanic and Atmospheric Administration iniciara en 2007 el proyecto HFIP (Hurricane Forecast Improvement Project). En el marco de este proyecto se desarrolló el modelo HWRF (Hurricane Weather Research and Forecasting Model), el cual presenta modificaciones en las parametrizaciones físicas que emplea para su uso específico en el pronóstico numérico de huracanes (Gall et al., 2013).

En investigaciones recientes se ha estudiado la influencia de las parametrizaciones en la habilidad de los modelos para representar los procesos de intensificación de los ciclones tropicales. Smith y Thomsen (2010) utilizaron el MM5 (Fifth-Generation Mesoscale Model) para estudiar la dependencia que existe entre la intensificación del ciclón tropical y la estructura cinemática de la capa límite, dependiendo de la parametrización de capa límite utilizada. En esta investigación se enfatiza el hecho de que la selección del esquema de parametrizaciones tiene un impacto importante sobre el cálculo de la intensidad realizado por el modelo. Por otro lado, numerosos estudios (Gopalakrishnan et al., 2011, 2012, 2013; Bao et al., 2012; Chen y Gopalakrishnan, 2015; Zhang and Marks, 2015) han empleado el HWRF para examinar el impacto de varias parametrizaciones en el pronóstico de intensidad.
Ko et al. (2017) desarrollaron una evaluación del campo de precipitación del HWRF para el huracán Harvey, con tres experimentos inicializados en diferentes horarios de pronóstico. Estos autores obtuvieron para este caso de estudio, que el modelo produce precipitación realista en términos de patrón, cantidad de lluvia, razón de lluvia radial y distribución de frecuencia. Sin embargo, en el núcleo de la convección se presentaron valores de razón de lluvia ligeramente mayor que las medidas. Por otra parte, Tran y Chiao (2013) evaluaron la precisión del campo de viento y de las estructuras de las bandas de lluvias con el HWRF para los sistemas Columbus Day (2011) y Fay (2008). Específicamente estudiaron el efecto de las parametrizaciones de microfísica de las nubes en los pronósticos para sistemas de rápida intensificación en términos de distribución de lluvia. Estos autores evidenciaron que ocurrió una subestimación de la precipitación para Columbus Day y una ligera sobrestimación para Fay, asociado a que un incremento del número de concentración de gotas en la configuración del modelo incrementa la distribución espacial de la precipitación.

Desde la década de los sesenta del Siglo XX, existen antecedentes en Cuba, en el INSMET (Instituto de Meteorología de Cuba), de la utilización de modelos de circulación atmosférica para el pronóstico numérico del tiempo y la trayectoria de los ciclones tropicales. Los primeros pasos fueron dados en colaboración con especialistas rusos mediante el empleo del modelo baroclínico adiabático de dos niveles desarrollado por Bortnikov (1968).

Para septiembre - octubre de 1998, Mitrani y Pérez (1999), realizaron los primeros experimentos de predicción del tiempo a corto plazo al paso de los huracanes "Georges", "Mitch", de sistemas frontales y de ondas tropicales mediante el empleo del modelo de pronóstico numérico del tiempo MM5.

Por otra parte, destaca la utilización del MM5 para el pronóstico de trayectoria de ciclones tropicales en investigaciones realizadas por Mitrani et al. (2002) y Mitrani y González (2005). Mitrani et al. $(2010,2011)$ plantean que este modelo mostró buena habilidad en el pronóstico de la trayectoria de huracanes, por períodos de hasta $48 \mathrm{~h}$, para zonas de Cuba y las Antillas Menores. A partir de 2011 se empieza a tener experiencia en el INSMET en el empleo del WRF (Weather Research and Forecasting Model). Mitrani et al. (2017) plantea que los resultados en el pro- 
nóstico de trayectoria fueron deficientes en aquellos plazos de tiempo superiores a las $48 \mathrm{~h}$.

Actualmente, el pronóstico numérico de ciclones tropicales en Cuba se efectúa a partir de las configuraciones del modelo WRF para condiciones medias de la atmósfera en los sistemas de pronóstico SPNOA (Sistema de Predicción Numérica Océano Atmósfera) (Pérez Bello et al., 2019) y SisPI (Sistema de Pronóstico Inmediato) (Sierra et al., 2015). Mitrani et al. (2019) evidenció que la componente atmosférica del SPNOA tiene habilidad para el pronóstico de trayectoria de ciclones tropicales en las primeras $48 \mathrm{~h}$ de pronóstico, sin embargo, entre las $48 \mathrm{y}$ $72 \mathrm{~h}$ los errores aumentan considerablemente. Este comportamiento está estrechamente relacionado con el hecho de que la habilidad de pronóstico en la predicción numérica se ve afectada por dos tipos de error: el error en las condiciones iniciales y el error propio del modelo relacionado con los métodos matemáticos para solucionar el sistema de ecuaciones, los métodos de interpolación empleados, los errores de truncamiento durante el cálculo numérico, entre otros (Nikki et al., 2013), el cual crece en la medida que temporalmente se aleja del instante de la inicialización. Adicionalmente, los algortimos de búsqueda del centro de la tormenta, tienen un impacto directo sobre el error de trayectoria e intensidad de los ciclones tropicales, el cálculo del viento máximo y de la presión mínima central del sistema.

Esto ha motivado que en esta investigación se plantee como objetivo comparar la habilidad de la componente atmosférica del sistema HWRF y la del modelo WRFHWRF para el pronóstico de intensidad y trayectoria, a través de simulaciones realizadas para el huracán Irma (2017), mediante el empleo de diferentes resoluciones horizontales.

\section{Materiales y Métodos}

\subsection{Modelos empleados}

2.1.1. Modelo WRF - NMM exportando seguimiento de vórtice (WRF - HWRF)

El NMM (Non-hydrostatic Mesoscale Model) es uno de los núcleos dinámicos del WRF, fue desarrollado por la NOAA y el NCEP (National Center for Environmental Prediction) de los Estados Unidos. Este núcleo dinámico incluye algoritmos computacionales para el cálculo del gradiente de presión, el parámetro de Coriolis, la divergencia de masa, esquemas de advección, procesos termodinámicos; así como un módulo no hidrostático.

Además, presenta la posibilidad de anidamiento de dominios y varias opciones de microfísica de las nubes, cúmulos, física de la capa superficial, capa fronteriza atmosférica, turbulencia y radiación atmosférica. La opción de seguimiento de vórtice se habilita exportando HWRF $=1$ al compilar y ejecutar el modelo. Una descrip- ción más detallada sobre aspectos técnicos fue desarrollada por Janjic et al. (2014).

\subsubsection{Modelo HWRF}

El HWRF es un modelo operativo para la predicción de huracanes en el NCEP. Fue desarrollado en conjunto por el EMC (Environmental Modeling Center) del NCEP, el GFDL (Geophysical Fluid Dynamics Laboratory) de la NOAA y el AOML (Atlantic Oceanographic and Meteorological Laboratory). Incluye la infraestructura del WRF y está basado en el núcleo dinámico NMM. Es un modelo acoplado océano-atmósfera con ecuaciones primitivas no hidrostáticas. Emplea un conjunto de parametrizaciones físicas desarrolladas para el pronóstico de ciclones tropicales. La inicialización del modelo incluye procedimientos de relocalización del vórtice ciclónico y de asimilación de datos.

A diferencia de otros modelos que se mantienen operativos en el NCEP durante todo el año, el HWRF es utilizado operacionalmente cuando el NHC o el JTWC (Joint Typhoon Warning Center) consideran que un disturbio atmosférico tiene condiciones para desarrollarse y convertirse en ciclón tropical (Holt et al., 2014 ; Biswas et al., 2017). En esta investigación solamente se empleó la componente atmosférica del HWRF.

\subsection{Caso de estudio}

Para la evaluación de las configuraciones de los modelos, se seleccionó el huracán Irma (Fig. 1) por la intensidad alcanzada, la permanencia como categoría 5 en la escala Saffir-Simpson por más de 24 h así como por los daños socioeconómicos provocados en las Islas del Caribe y los Estados Unidos. Solo en Cuba provocó la pérdida de 9 vidas humanas, considerables pérdidas materiales entre ellas: más de 150000 casas sufrieron daños (15 000 destrucción total) y 466 granjas avícolas destruidas. El total de daños económicos en el país caribeño fue valorado en 200 millones USD (Cangialosi et al., 2018).

\subsection{Diseño de los experimentos}

Las corridas realizadas se inicializaron en todos los casos a las 00 UTC con las salidas de pronóstico del GFS (Global Forecast System) a 0.5 grados de resolución obtenidas del NCDC (U.S National Climatic Data Center ) Se realizaron corridas desde el 1ro hasta el 12 de septiembre de 2017 para un plazo de pronóstico de $72 \mathrm{~h}$. Las condiciones de frontera se actualizaron cada $6 \mathrm{~h}$ y el paso temporal de integración fue de $69 \mathrm{~s}$ para el dominio de $27 \mathrm{~km}$ y de $30 \mathrm{~s}$ para el de $18 \mathrm{~km}$ (Holt et al., 2014).

Por otro lado se adaptó el algoritmo que viene incorporado en el código fuente del sistema HWRF, para detectar la posición $i, j$ del dominio interno en el dominio exterior a partir del centro de la tormenta. Además, fue diseñado un procedimiento computacional para la visualización de las salidas del modelo. 


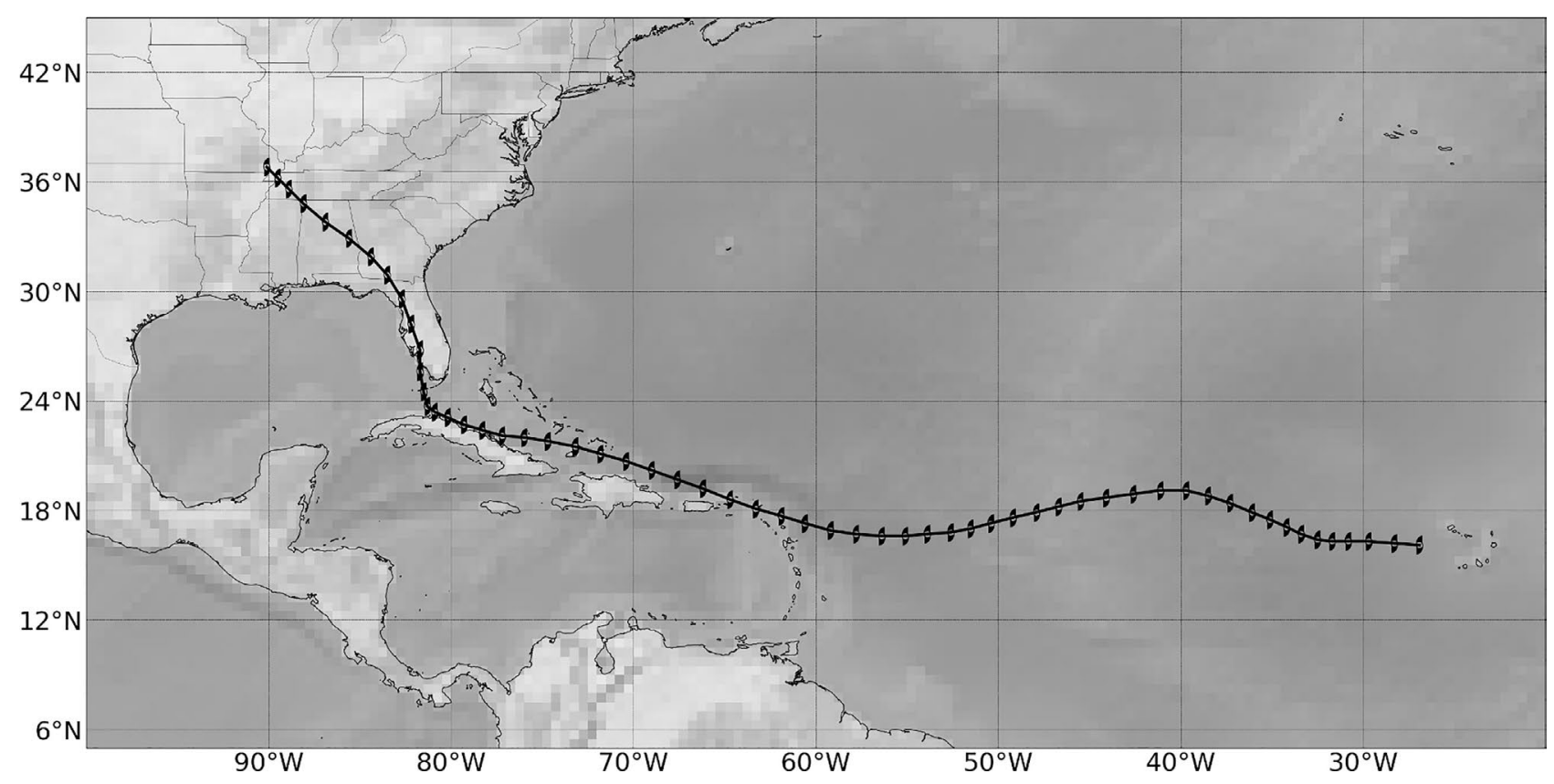

Figura 1 - Trayectoria del Huracán Irma (30 de agosto - 12 de septiembre de 2017).

Para la determinación del centro y la intensidad de la tormenta se emplearon las variables TRACKER_FIXLAT, TRACKER_FIXLON, TRACKER_PMIN y TRACKER_VMAX que están disponibles en las salidas de ambos modelos.

\subsubsection{Parametrizaciones fisicas empleadas}

Las parametrizaciones juegan un papel importante en los sistemas de pronóstico numérico del tiempo, influyendo directamente en la precisión de los pronósticos, en la capacidad de cómputo requerida y en su tiempo de ejecución.

En la Tabla 1 se muestran los aspectos fundamentales de las configuraciones empleadas. Se tuvieron en cuenta las recomendaciones que realiza Janjic et al. (2014) para el pronóstico de ciclones tropicales mediante el
WRF-HWRF, mientras que en la componente atmosférica del HWRF, se empleó la configuración descrita por Biswas et al. (2017) para las corridas operativas del mismo en el NCEP, durante la temporada ciclónica del 2017.

Las parametrizaciones de microfísica de nubes empleadas fueron la de Ferrier modificada para los trópicos (Ferrier et al., 2002) para el WRF-HWRF y FerrierAligo (Aligo et al. 2017) para la componente atmosférica del HWRF. Esta última es una modificación de Ferrier tropical que adiciona una representación de la concentración máxima de hielo en función de la temperatura. Incluye también un aumento de la retrodispersión de radar para la humedad y cuando la temperatura es inferior a cero grados celsius, la lluvia y el hielo coexisten en una intensa corriente ascendente. Además incorpora un algoritmo de producción de hielo en la nube y una reducción de la velocidad de caída del rimed-ice.

Tabla 1 - Configuraciones empleadas en los modelos.

\begin{tabular}{lcc}
\hline Aspectos & WRH-HWRF & HWRF \\
\hline Sistema de ecuaciones & Ecuaciones Primitivas con opción no hidrostática & Ecuaciones Primitivas con opción no hidrostática \\
Resolución vertical & 32 niveles verticales & 32 niveles verticales \\
Parametrización de radiación de onda larga & Modified GFDL & RRTMG \\
Parametrización de radiación de onda corta & Modified GFDL & RRTMG \\
Parametrización de cúmulo & Simplified Arakawa-Schubert (SAS) & Scale-Aware Simplified Arakawa-Schubert (SASAS) \\
Parametrización de microfísica & Ferrier modificada para HWRF & Ferrier-Aligo \\
Capa Fronteriza Atmosférica & NCEP Global Forecasting System & GFS Hybrid-EDMF PBL \\
Procesos de superficie subyacente & GFDL surface layer & HWRF surface layer \\
Seguimiento de vórtice & GFDL vortex tracker & GFDL vortex tracker \\
Time step & $69 \mathrm{~s}$ & $69 \mathrm{~s}$ \\
\hline
\end{tabular}


Por su parte, en el WRF-HWRF emplea el esquema simplificado Arakawa-Schubert (SAS) para representar los procesos convectivos (Han y Pan, 2011) mientras en el HWRF se emplea el esquema simplificado ArakawaSchubert independiente de la escala (SASAS), que es una modificación de SAS desarrollada por Han et al. (2017). En esta versión la convección profunda y el flujo de masa en la base de la nube es una función de la velocidad media de la corriente ascendente cuando la resolución horizontal es menor a $8 \mathrm{~km}$, en lugar de derivarse de la suposición de cuasi equilibrio de Arakawa-Schubert (Arakawa y Schubert, 1974), mientras que para convección poco profunda, el flujo de masa de nube es una función de la velocidad media de la corriente ascendente, en lugar de una función de la escala de velocidad convectiva.

En el caso de la representación de la capa fronteriza atmosférica (PBL, según sus siglas en inglés), se empleó en el WRF-HWRF el esquema NCEP GFS (Hong y Pan, 1996), que es un esquema de difusión vertical de primer orden que utiliza el enfoque de Richardson para estimar iterativamente la altura de PBL comenzando desde el suelo. Por su parte, en la componente atmosférica del HWRF se empleó el esquema GFS Hybrid-EDMF (Biswas et al., 2017) donde la mezcla no local en condiciones convectivas está representada por un enfoque de flujo de masa.

Para una mayor información y descripción de las parametrizaciones empleadas se debe consultar Janjic et al. (2014) y Biswas et al. (2017).

\subsection{Dominios de cómputo}

El área para el estudio fue la cuenca del Atlántico Norte y para los experimentos se configuraron dos dominios anidados interactivos bidireccionales con $27-9 \mathrm{~km}$ y 18-6 km de resolución como se muestra en la Fig. 2. A partir de ello se determinaron 8 configuraciones, en 4 de ellas el dominio exterior se localizó en el centro de la tormenta y en las restantes en un punto fijo de latitud y longitud (ver Tabla 2). El dominio interno se movió siguiendo el centro de la tormenta durante el período de integración del modelo.

\subsection{Estaciones meteorológicas y boyas empleadas para la verificación}

Para la verificación se emplearon los registros de 20 boyas del NDBC (U. S. National Data Buoy Center) perteneciente a la NOAA. Los datos de las mismas fueron obtenidos del sitio web del NDBC. Se emplearon los datos de 36 estaciones de superficie pertenecientes a la Red Nacional de Estaciones Meteorológicas de Cuba. Los registros de las estaciones cubanas fueron adquiridos en el archivo del Centro del Clima del INSMET. Además, se empleó la base de datos HURDAT2, de la cual se extrajeron la posición y la intensidad del huracán Irma para cada plazo de tiempo.

En la Fig. 3 se observa la distribución espacial de las mismas. Para la selección de estas estaciones se tuvo en cuenta que su ubicación geográfica estuviera cercana a la

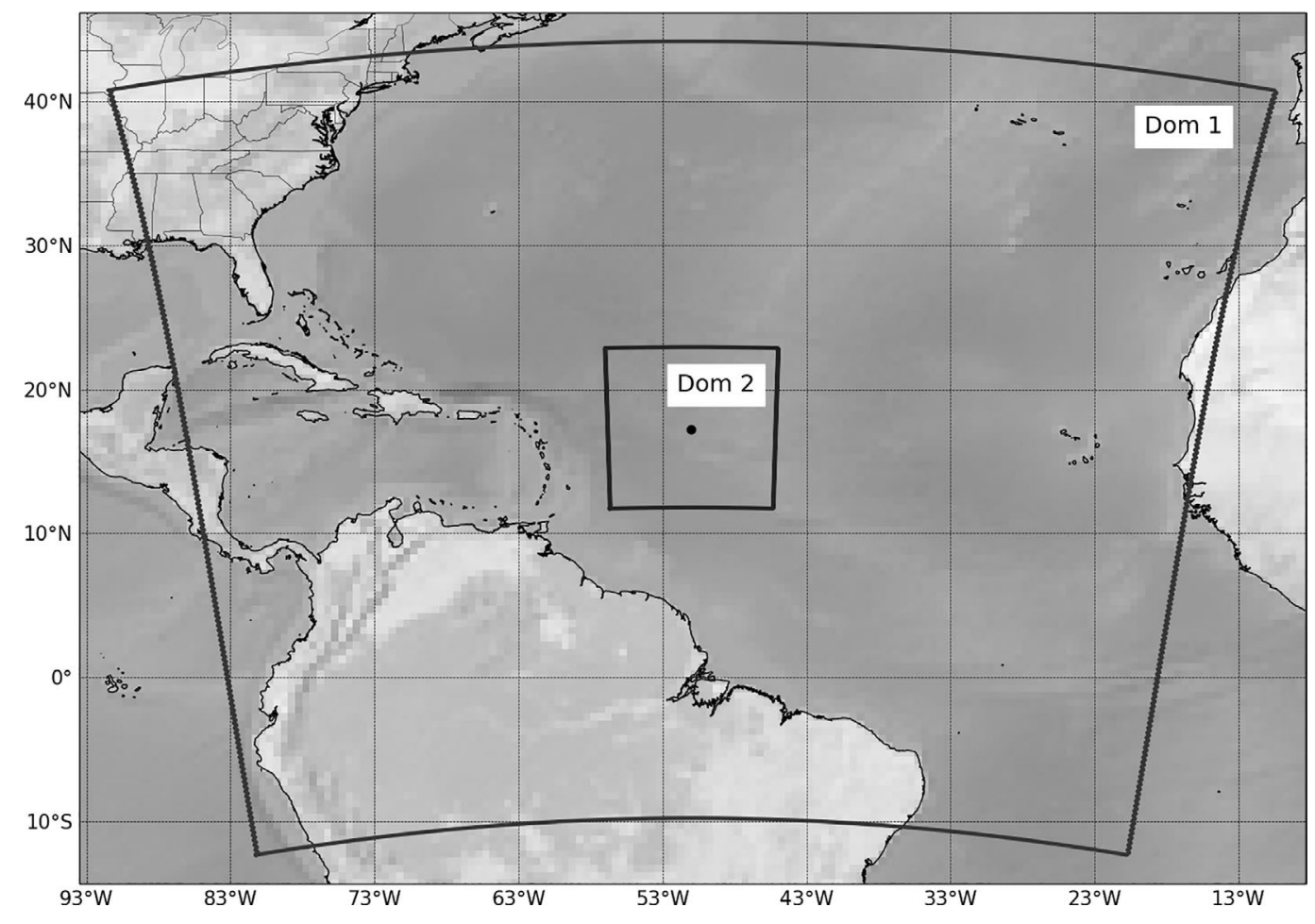

Figura 2 - Dominios de cómputo. 
Tabla 2 - Configuración de los dominios de cómputo utilizados.

\begin{tabular}{llccr}
\hline No. & \multicolumn{1}{c}{ Configuraciones } & Dominio $1(\mathrm{~km})$ & Dominio 2 $(\mathrm{km})$ & Ubicación \\
\hline 1 & HWRF (27-9) & 27 & 9 & $22^{\circ} \mathrm{N}, 60^{\circ} \mathrm{W}$ \\
2 & HWRF (27-9-m) & 27 & 9 & Centro de la tormenta \\
3 & HWRF (18-6) & 18 & 6 & $22^{\circ} \mathrm{N}, 60^{\circ} \mathrm{W}$ \\
4 & HWRF (18-6-m) & 18 & 6 & Centro de la tormenta \\
5 & WRF - HWRF (27-9) & 27 & 9 & $22^{\circ} \mathrm{N}, 60^{\circ} \mathrm{W}$ \\
6 & WRF - HWRF (27-9-m) & 27 & Centro de la tormenta \\
7 & WRF - HWRF (18-6) & 18 & 6 & $22^{\circ} \mathrm{N}, 60^{\circ} \mathrm{W}$ \\
8 & WRF - HWRF (18-6-m) & 18 & 6 & Centro de la tormenta \\
\hline
\end{tabular}

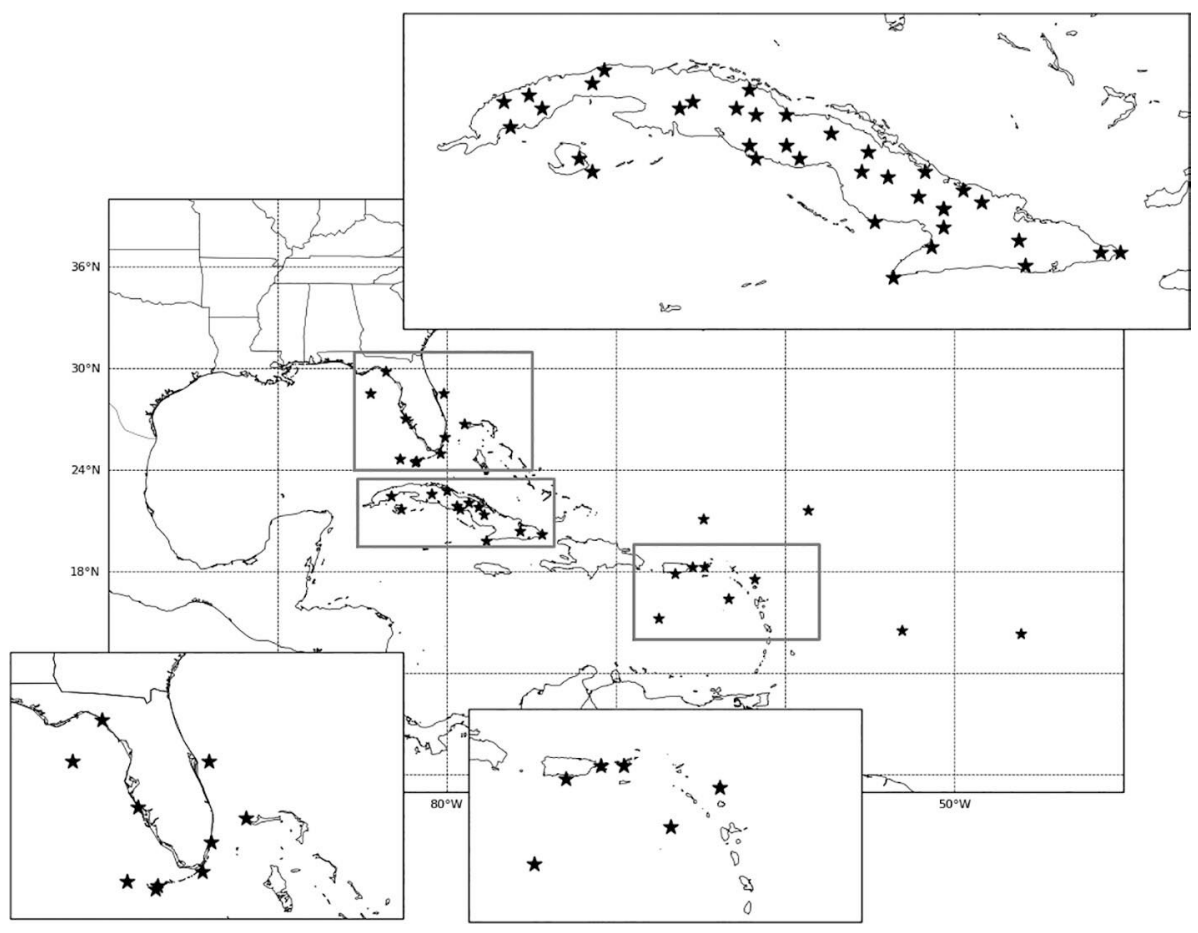

Figura 3 - Ubicación espacial de las estaciones utilizadas para la verificación.

trayectoria descrita por el huracán Irma así como, que existiera disponibilidad de series completas de datos en el período objeto de estudio.

\subsection{Estadígrafos empleados para la verificación}

\subsubsection{Coeficiente de Nash-Sutcliffe}

El criterio de Nash y Sutcliffe (1970) es uno de los más usados en Hidrología para evaluar la eficiencia de las simulaciones realizadas. Teniendo en cuenta que en su formulación se trabaja con valores simulados y valores observados de determinada propiedad cuantificable, en esta investigación se usó este criterio como una medida de la habilidad de las configuraciones empleadas en los modelos para simular el comportamiento real de las variables meteorológicas. El criterio se define como:

$$
\mathrm{NS}=1-\frac{\sum\left(x_{i}-y_{i}\right)^{2}}{\sum\left(y_{i}-\overline{y_{i}}\right)^{2}}
$$

donde $n$ es el total de datos, $x_{i}$ son los valores simulados, $y_{i}$ son los valores observados y $\overline{y_{i}}$ es la media de los valores observados. Si la simulación es perfecta NS $=1$. Un criterio de evaluación se expone en la Tabla 3.

\subsubsection{Error absoluto medio}

Ofrece una medida de cuanto se alejan los valores simulados de los observados. Se define como:

$$
\text { MAE }=\frac{\sum\left|x_{i}-y_{i}\right|}{n}
$$

Entre más cercanos se encuentren los valores de MAE a cero, más precisa es la simulación. 
Tabla 3 - Valores referenciales del criterio de Nash-Sutcliffe.

\begin{tabular}{ll}
\hline NS & Criterio \\
\hline$<0.2$ & Insuficiente \\
$0.2-0.4$ & Satisfactorio \\
$0.4-0.6$ & Bueno \\
$0.6-0.8$ & Muy bueno \\
$>0.8$ & Excelente \\
\hline
\end{tabular}

\subsubsection{BIAS}

El BIAS proporciona la diferencia entre el valor estimado y el valor real:

$$
\text { BIAS }=\frac{\sum\left(x_{i}-y_{i}\right)}{n}
$$

Entre más cercanos se encuentren los valores del BIAS a cero, más precisa es la simulación.

\subsection{Parámetros computacionales del clúster de cálculo}

Las simulaciones se realizaron en el clúster de cálculo Benjamin del Departamento de Meteorología del Instituto Superior de Tecnologías y Ciencias Aplicadas de la Universidad de La Habana. El clúster tiene sistema operativo Ubuntu 14.04, con 5 nodos de cómputo Intel Core i5 de 4 procesadores físicos y $8 \mathrm{~Gb}$ de memoria RAM cada uno.

\section{Resultados y Discusión}

\subsection{Pronóstico de trayectoria}

En la Fig. 4a se puede observar como los errores durante las primeras $48 \mathrm{~h}$ en el pronóstico de trayectoria para todas las configuraciones se encuentran en el mismo orden que el error cometido por el NHC en su pronóstico oficial en la temporada ciclónica del 2016. De forma general, los errores en el pronóstico de la trayectoria oscilan entre los $27 \mathrm{~km}$ para las primeras $6 \mathrm{~h}$ hasta los $200 \mathrm{~km}$ para las 72 h. Cangialosi et al. (2018) plantea que el error medio cometido en el pronóstico oficial del NHC para el huracán Irma disminuyó entre un 30 y 40\% con respecto a los errores medios oficiales de los 5 años anteriores para todos los períodos de pronóstico.

En la Fig. 4b se observa como los errores medios en las primeras $24 \mathrm{~h}$ de pronóstico para todas las configuraciones, presentan una disminución entre 5 y $30 \%$ comparados con los errores medios oficiales de los últimos 5 años. Sin embargo, para todas las configuraciones estudiadas se observó en las últimas horas de pronóstico (6072 h) un aumento del error, siendo máximo con un $43 \%$ para la configuración HWRF 18-6-m. Es preciso señalar que de forma general todas las configuraciones estudiadas presentaron un comportamiento similar.

Al analizar la diferencia entre el error de cada configuración con respecto al error medio del pronóstico oficial del NHC para el huracán Irma (Fig. 5) se comprueba que la configuración que presenta los mejores resultados es HWRF 18-6-m, aunque en los últimos pla-

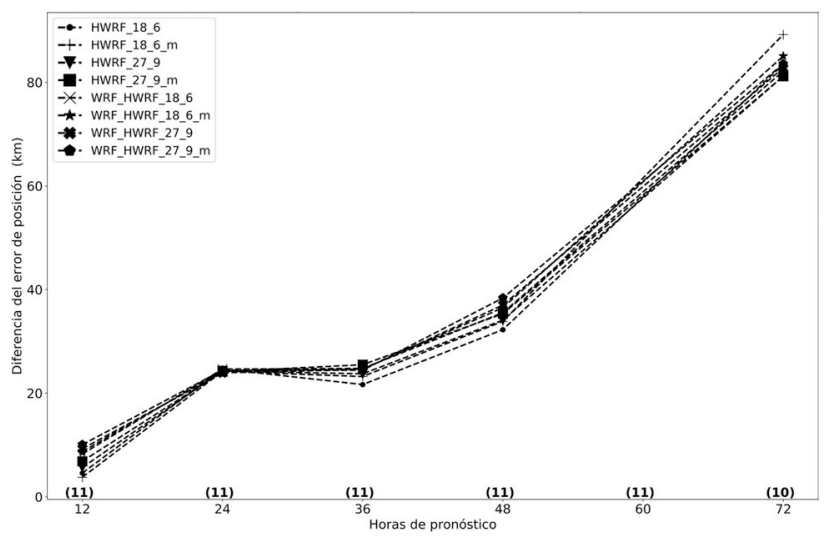

Figura 5 - Diferencia del error de cada configuración con respecto al error medio del NHC del 2016.
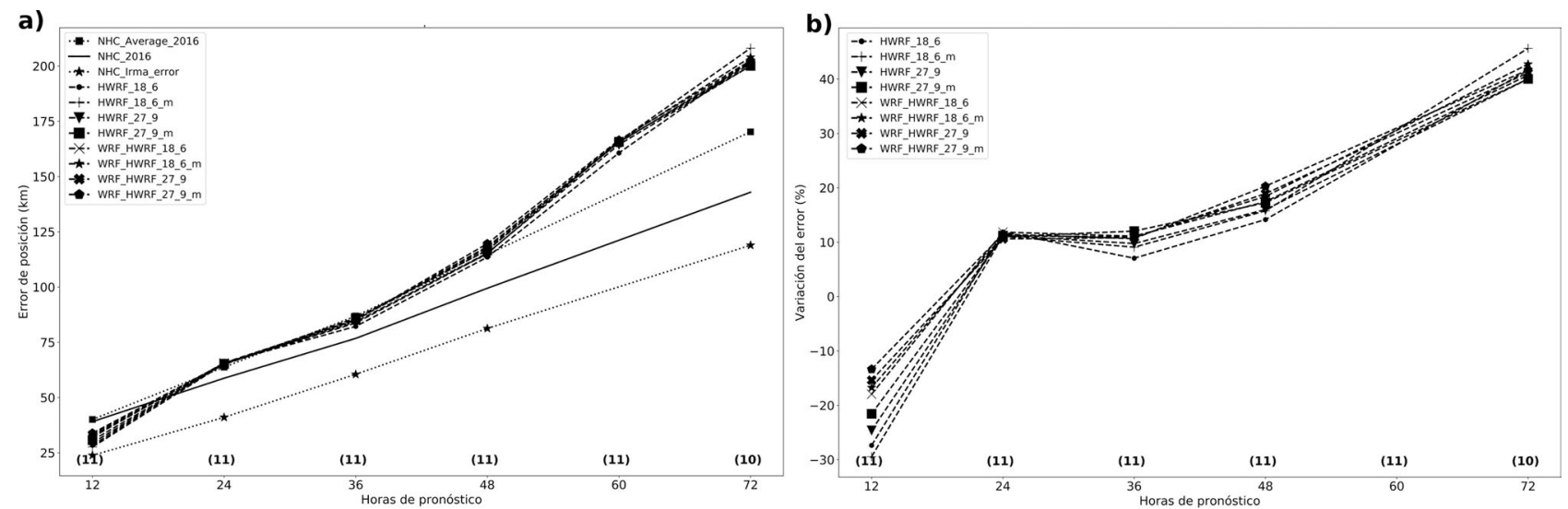

Figura 4 - a) Error de posición en el pronóstico de la trayectoria para cada configuración. b) Disminución del error en \% respecto al error medio del NHC en el período 2012-2016 (NHC Average 2016 representa al error medio del NHC en el período 2012-2016). 
zos de tiempo muestra un incremento poco notable del error medio.

En la Fig. 6 se muestran las trayectorias simuladas con las ocho configuraciones estudiadas para las corridas inicializadas el 7 y 9 de septiembre de 2017 a las 00 UTC respectivamente. Es apreciable como en los primeros plazos de pronóstico existe un agrupamiento de las curvas alrededor de la trayectoria real del sistema, mientras que en los plazos finales de tiempo existe una mayor dispersión de las trayectorias. Lo anterior está en correspondencia con los errores medios de las configuraciones representados en la Fig 4a-b. En ambas simulaciones se observa como las trayectorias pronosticadas por las ocho configuraciones describen un comportamiento similar al pronóstico realizado por los modelos operativos del NCEP.

El comportamiento observado en los errores en el pronóstico de trayectoria está directamente relacionado con el hecho de que los modelos de pronóstico indicaban una profundización de la vaguada situada sobre el continente americano y como resultado, un debilitamiento de la cuña anticiclónica (Fig. 7). Este proceso generaría un giro en la trayectoria del huracán Irma hacia el oeste-no-
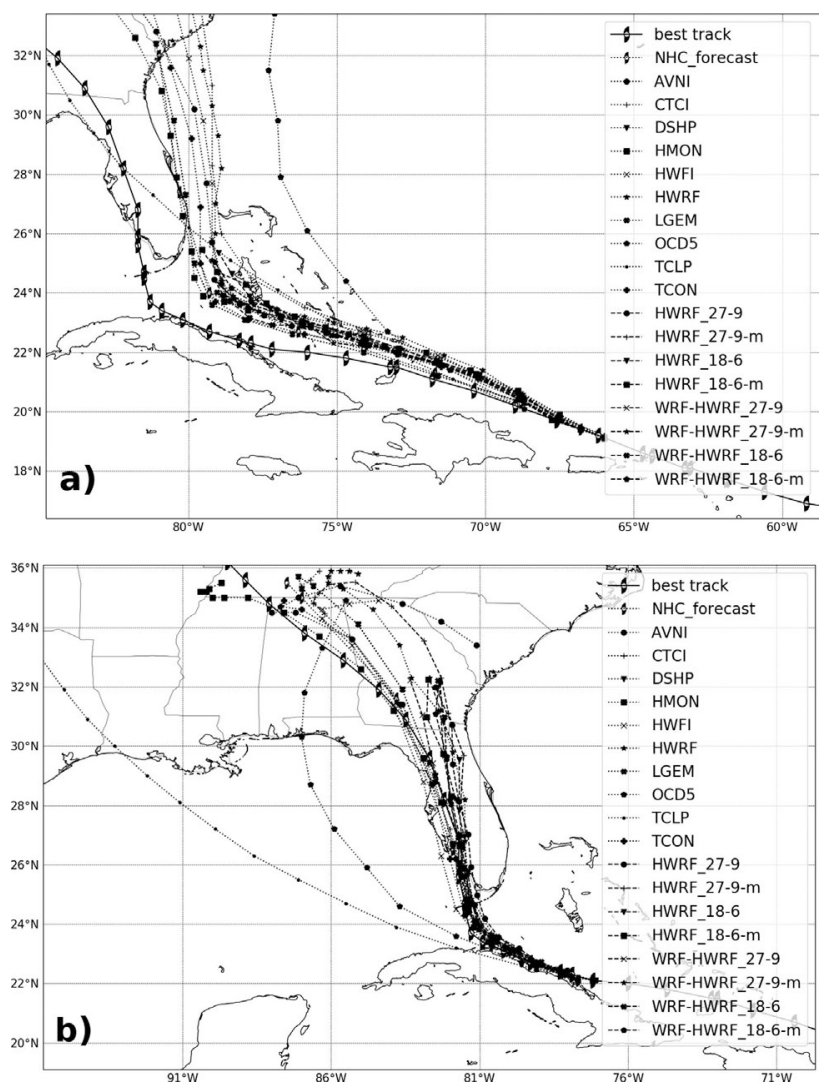

Figura 6 - Trayectoria pronosticada con las 8 configuraciones para el huracán Irma en la corrida inicializada el día a) 7 de septiembre de 2017 a las 00:00 UTC, b) 9 de septiembre de 2017 a las 00:00 UTC. Se han representado las trayectorias correspondientes al pronóstico oficial del NHC, a modelos operativos en el NCEP y el NWS (U. S. National Weather Service) y la best track del NHC. roeste sobre los mares al norte del centro de Cuba (Fig. 7a, Fig. 7b) llevando al huracán hacia la costa oriental de la Florida. Esta situación no ocurrió en la realidad, el sistema transitó paralelo a la costa norte cubana hasta cambiar su movimiento, al norte de Matanzas, hacia la costa occidental de la Península de la Florida (Fig. 7c-d).

\subsection{Pronóstico de intensidad}

Cangialosi et al. (2018) plantea que los errores en el pronóstico oficial de intensidad realizado en el NHC para el huracán Irma fueron superiores para todos los plazos de tiempo que los errores medios en los últimos 5 años. Mientras que los del modelo estadístico y de persistencia DSHIFOR5 (Decay Statistical Hurricane Intensity Forecast) fueron notablemente superiores en el mismo período. La intensidad de Irma fue más difícil de pronosticar que la de un ciclón tropical típico. Una de las características más llamativas y difícil de pronosticar del sistema tropical fueron los ciclos de intensificación rápida que experimentó durante su trayectoria de vida.

\subsubsection{Velocidad máxima del viento}

El pronóstico de la velocidad máxima del viento presenta un comportamiento atípico, pues se observa una disminución del error a medida que aumentan los plazos de pronóstico. Esto se debe fundamentalmente al tiempo que requiere el modelo para la estabilización de los campos meteorológicos. Los errores oscilan entre los $75 \mathrm{~km} / \mathrm{h}$ para las primeras $6 \mathrm{~h}$ hasta los $50 \mathrm{~km} / \mathrm{h}$ a las $72 \mathrm{~h}$ (Fig. 8). Estos valores se encuentran muy por encima de los errores medios del NHC para el período 2012-2016. Esta diferencia se atribuye fundamentalmente a la no relocalización del vórtice y a los necesarios reajustes de los campos meteorológicos. En todos los plazos de tiempo ocurre un subestimación de la velocidad máxima del viento alcanzada por el huracán Irma. Las configuraciones que presentaron los mejores resultados fueron WRF-HWRF 18-6 y WRF-HWRF 18-6-m.

\subsubsection{Presión mínima central}

En cuanto a la presión mínima central, los errores oscilaron entre los $25 \mathrm{hPa}$ para las primeras horas hasta $14 \mathrm{hPa}$ en las $72 \mathrm{~h}$ (Fig. 9). En todos los plazos de tiempo se sobrestima la presión mínima central alcanzada por el huracán Irma. Las configuraciones que presentaron los mejores resultados fueron HWRF 18-6-m y HWRF 18-6.

En resumen, en el pronóstico de intensidad se observan los mayores errores en los primeros plazos de tiempo. Este comportamiento está directamente relacionado con la no utilización de un esquema de relocalización de vórtice en la inicialización de los modelos y al hecho de que las salidas pronóstico del GFS muestran una representación débil del huracán Irma, comparado con los reportes del NHC. 

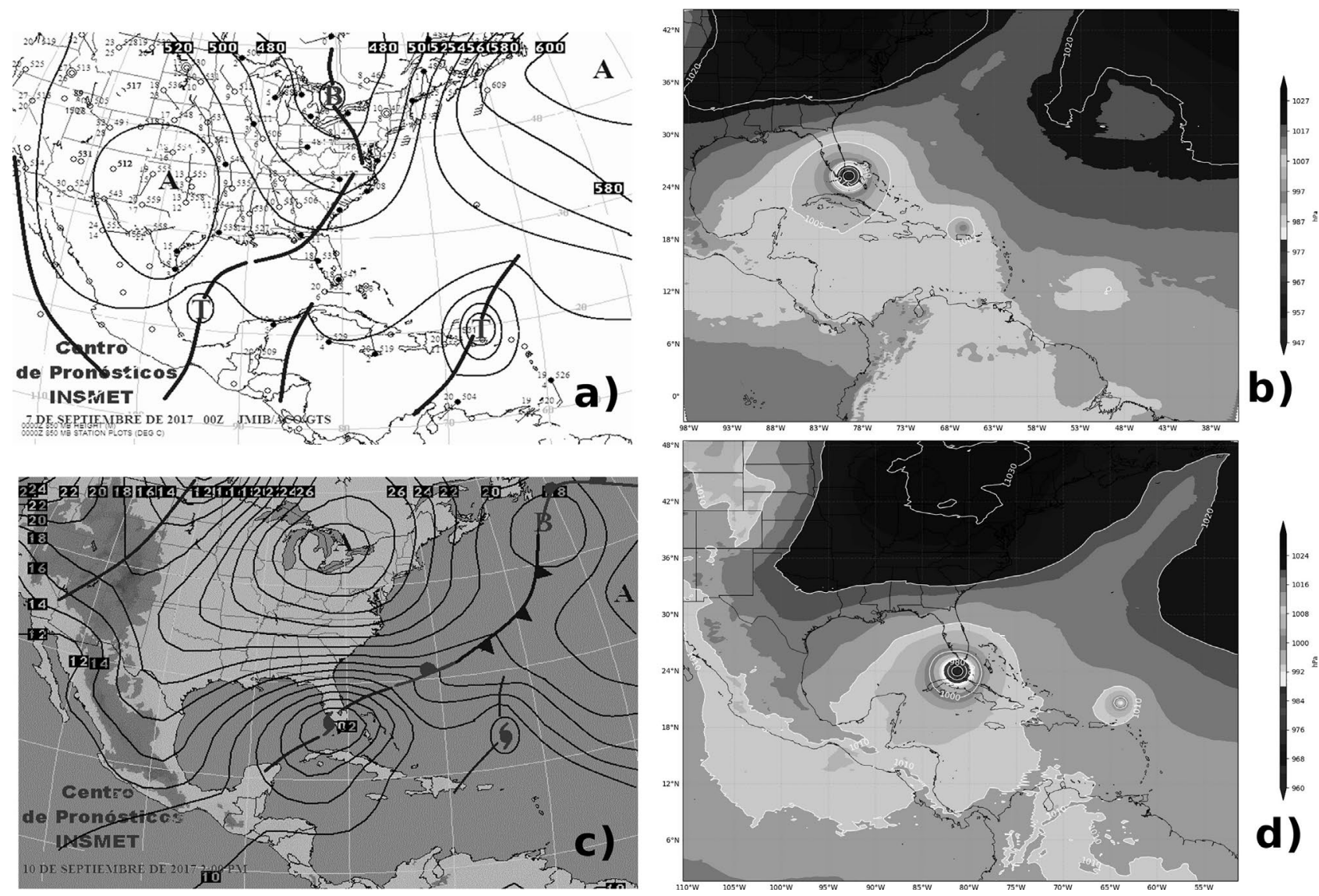

Figura 7 - Situación sinóptica a) mapa de $850 \mathrm{hPa}$ del INSMET para el 7 de septiembre de 2017 a las 00 UTC, b) mapa de superficie para el 10 de septiembre a las 06 UTC a partir de la inicialización del 7 de septiembre 2017 a las 0000 UTC, c) mapa de superficie del INSMET para el 10 de septiembre de 2017 a las 18 UTC, d) mapa de superficie para el 10 de septiembre a las 06 UTC a partir de la inicialización del 10 de septiembre 2017 a las 0000 UTC

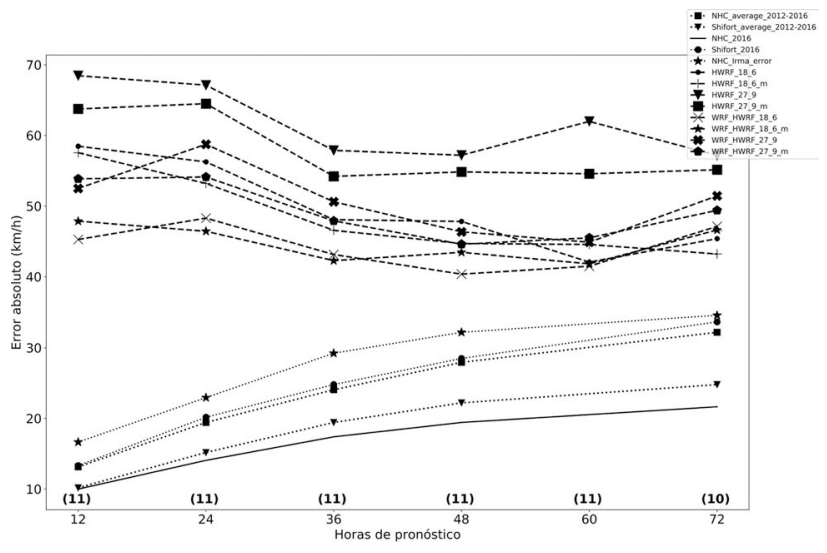

Figura 8 - Error en el pronóstico de velocidad máxima del viento.

\subsection{Análisis de los cambios de intensidad}

Según Cangialosi et al. (2018) el huracán Irma presentó un ciclo de rápida intensificación del 4 de septiembre a las 18:00 UTC al 5 de septiembre a las 12:00 UTC. En apenas $18 \mathrm{~h}$ el sistema aumentó la velocidad del viento de $210 \mathrm{~km} / \mathrm{h}$ a $275 \mathrm{~km} / \mathrm{h}$ y disminuyó su presión central de 944 a $929 \mathrm{hPa}$. El comportamiento descrito anteriormente

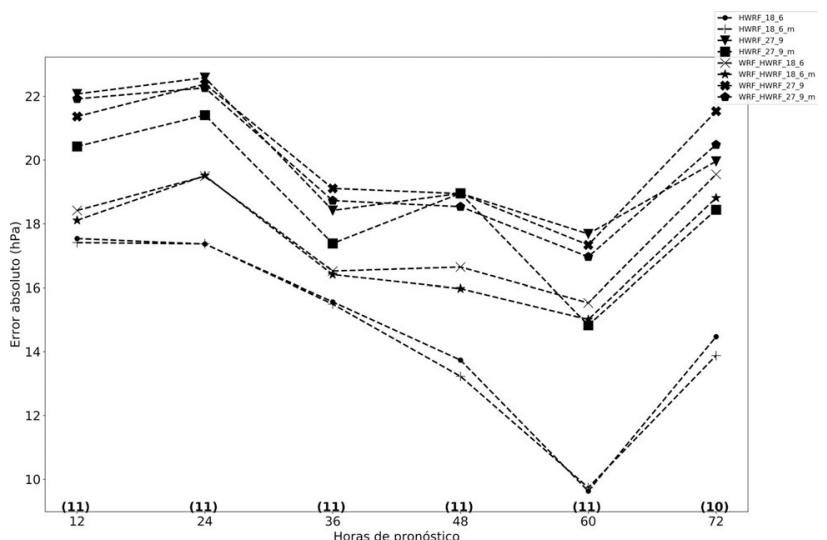

Figura 9 - Error en el pronóstico de la presión mínima central.

se observa en la Fig. 10a. Este cambio brusco de intensidad no fue pronosticado por los modelos operativos en el NCEP y tampoco por las configuraciones analizadas en esta investigación, aunque es notable que en todos los casos se observó una paulatina intensificación del sistema. Destaca como las configuraciones objeto de estudio pronosticaron velocidades del viento inferiores a las calcula- 

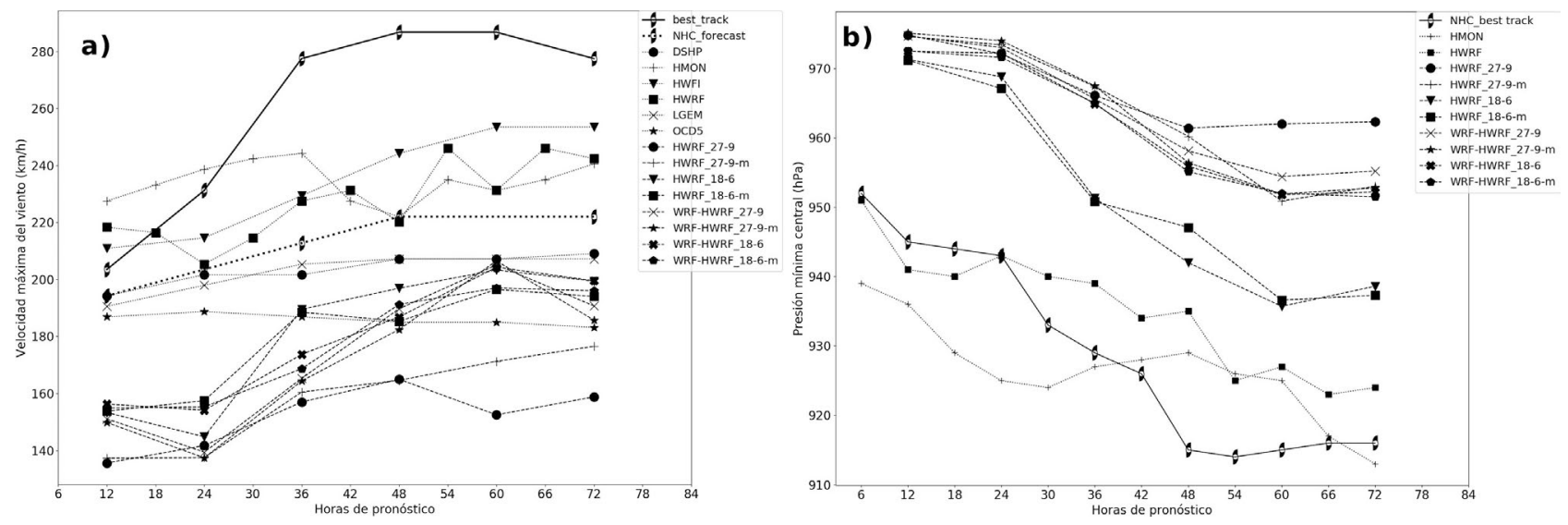

Figura 10 - Pronóstico de intensidad para el huracán Irma en la corrida inicializada el 4 de septiembre de 2017 a las 00:00 UTC a) Velocidad máxima del viento, b) Presión mínima central. Se han representado las intensidades pronosticadas correspondientes al pronóstico oficial del NHC, a modelos operativos en el NCEP y la best track.

das por más del $80 \%$ de los modelos operativos en el NCEP.

Para el caso de la presión mínima central (Fig. 10b), se puede resumir que la habilidad de las configuraciones analizadas para representar el cambio de presión fue deficiente. Sin embargo, las configuraciones HWRF 18-6 y HWRF 18-6-m describieron una variación de la presión de $20 \mathrm{hPa}$ en $24 \mathrm{~h}$. Este comportamiento fue similar al que sufrió el huracán Irma, aunque los valores simulados estuvieron aproximadamente $20 \mathrm{hPa}$ por encima del valor real de presión mínima que alcanzó el sistema.

\subsection{Comparación con datos de estaciones}

Para el análisis general de las variables presión atmosférica y velocidad del viento en los puntos de estaciones se interpolaron los campos del modelo a las coordenadas de las estaciones.

\subsubsection{Velocidad del viento}

En la Fig. 11 se observa como el error absoluto medio de la fuerza del viento en las coordenadas de las boyas es inferior a $3.8 \mathrm{~m} / \mathrm{s}(13 \mathrm{~km} / \mathrm{h})$ para todas las configuraciones. La configuración HWRF 18-6-m es la que presenta los mejores resultados con un error absoluto inferior a $3.3 \mathrm{~m} / \mathrm{s}(12 \mathrm{~km} / \mathrm{h})$. Para el coeficiente de correlación los valores oscilaron alrededor de 0.6 lo cual es un indicativo de la similitud de la evolución temporal de la fuerza del viento simulada por el modelo y los valores registrados en las estaciones.

Un análisis del coeficiente de Nash - Sutcliffe y partiendo del criterio establecido en la Tabla 3, se comprobó que para $12 \mathrm{a} 24 \mathrm{~h}$ de simulación el valor pronosticado de la velocidad del viento en las coordenadas de las estaciones es satisfactorio. Para las $30 \mathrm{~h}$ existe una disminución brusca del valor de NS y a partir de las 36 h los valores oscilaron alrededor de cero, lo cual cataloga a la simulación como insuficiente. Un comportamiento similar

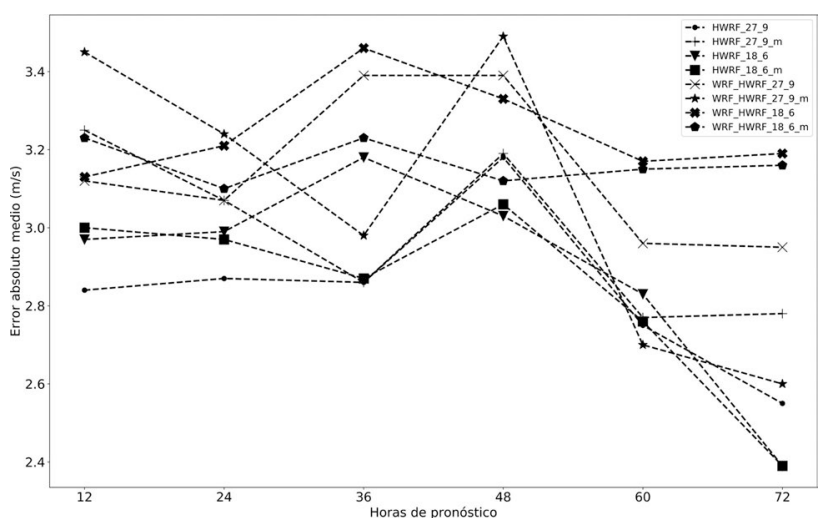

Figura 11 - Error absoluto medio en la fuerza del viento de todas las configuraciones al comparar el valor simulado en las coordenadas de cada estación con el valor real registrado.

puede observarse al analizar el comportamiento de la raíz del error cuadrático medio para cada plazo de tiempo. Es notable como el valor de la raíz del error cuadrático medio oscila entre 3 y $5 \mathrm{~m} / \mathrm{s}$ de forma general, con un máximo global de $5.5 \mathrm{~m} / \mathrm{s}$ para las $30 \mathrm{~h}$.

A partir del análisis anterior es apreciable como la configuración HWRF 18-6-m fue la que presentó los mejores resultados en el pronóstico de la fuerza del viento en los puntos de estaciones analizados.

\subsubsection{Presión atmosférica}

En la Fig. 12 se observa como el error absoluto medio de la presión atmosférica en las coordenadas de las estaciones es inferior a $3 \mathrm{hPa}$ para todas las configuraciones. Para el coeficiente de correlación los valores oscilaron alrededor de 0.90 lo que demuestra que la evolución temporal de la presión pronosticada tiene un comportamiento similar a la evolución temporal real de esta variable. 


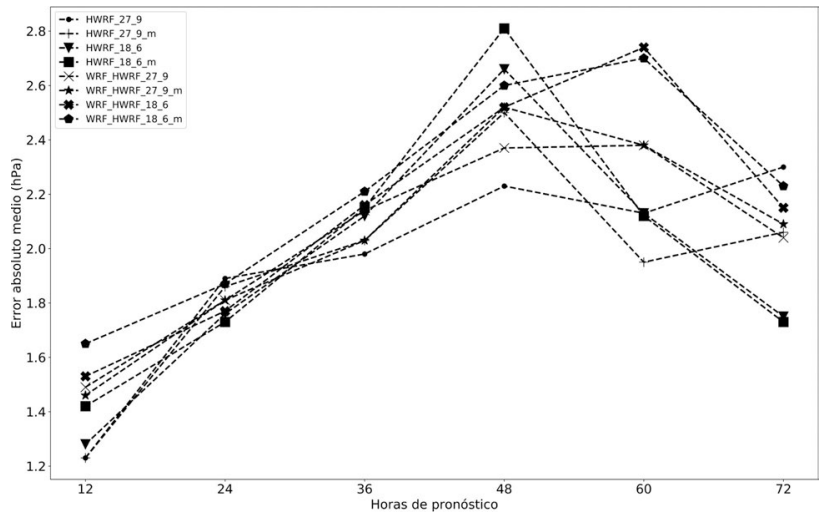

Figura 12 - Error absoluto medio en la presión atmosférica de todas las configuraciones al comparar el valor simulado en las coordenadas de cada estación con el valor real registrado.

Al analizar el coeficiente de Nash - Sutcliffe se observó como los valores del mismo están alrededor de 0.7 para las primeras $48 \mathrm{~h}$ de pronóstico, lo cual evidencia la eficiencia del modelo para el pronóstico de la presión atmosférica. El estudio de la raíz del error cuadrático medio arroja que los valores simulados por las configuraciones de los modelos se desvían de los valores reales en una magnitud que oscila entre 1.5 y $4.5 \mathrm{hPa}$. De un análisis general se determinó que la configuración HWRF 27-9 fue la que presentó los mejores resultados en el pronóstico de la presión atmosférica.

\subsection{Resumen del pronóstico de trayectoria e intensidad de las configuraciones}

Aunque ambos modelos emplean parametrizaciones similares, la habilidad de las mismas para representar los fenómenos de subrejilla como el física de las nubes y los flujos turbulentos difiere en ambos sistemas (Janjic et al., 2014; Biswas et al., 2017). Esta característica influye considerablemente en los errores observados anteriormente.

Por otro lado, la precisión de las condiciones de frontera juega un importante papel en el pronóstico numé- rico del tiempo, porque las ondas atmosféricas y las perturbaciones generadas en la frontera pueden propagarse rápidamente por todo el dominio (Sinhg y Mandal, 2014). Por lo anterior, los errores observados en la habilidad de ambos modelos para el pronóstico de la intensidad y trayectoria del huracán Irma, están influenciados indirectamente por la resolución temporal con la que se actualizaron las condiciones de frontera en los experimentos desarrollados.

A partir de las consideraciones anteriores y del análisis de los estadígrafos: Nash-Sutcliffe, error absoluto medio, raíz del error cuadrático medio y coeficiente de correlación de Pearson, así como de los errores medios determinados en el pronóstico de intensidad y trayectoria; se estableció una escala de la habilidad de cada configuración para el pronóstico fiable de la evolución temporal de sistema ciclónico tropical. Los resultados del análisis se muestran en la Tabla 4.

Se aprecia como la configuración HWRF 18-6-m es la que presenta el mejor comportamiento en más del $60 \%$ de los parámetros analizados. Este resultado está estrechamente relacionado con el hecho de que el empleo de una mayor resolución horizontal y una descripción física más detallada permite una mejor descripción de la estructura interna de la tormenta. Esto garantiza mayor habilidad en el pronóstico de intensidad y trayectoria.

\subsection{Análisis del tiempo de corrida}

El tiempo de corrida promedio para cada configuración según el diseño de los experimentos (epígrafe 2.3) se obtuvo en el clúster de cálculo Benjamin (epígrafe 2.7). En la Tabla 5 se muestra el tiempo de cómputo de cada configuración para un plazo de pronóstico de $72 \mathrm{~h}$. Es apreciable como la configuración HWRF 18-6-m, que ha presentado el mejor comportamiento en la evaluación realizada tiene un tiempo de corrida cercano a las $4 \mathrm{~h}$, lo cual puede ser una limitante para su implementación en el pro-

Tabla 4 - Determinación de la habilidad de cada configuración para el pronóstico de la trayectoria y la intensidad. COR: Coeficiente de correlación de Pearson NS: Coeficiente de Nash-Sutcliffe MAE: Error Absoluto Medio RMSE: Raíz del error cuadrático medio RF: Ranking Final Vmax: Velocidad máxima del viento Pmin: Presión mínima central V: Velocidad del viento P: Presión Atmosférica.

\begin{tabular}{|c|c|c|c|c|c|c|c|c|c|c|c|c|}
\hline \multirow[t]{2}{*}{ Configuraciones } & \multirow[t]{2}{*}{ Track } & \multicolumn{2}{|c|}{ Intensidad } & \multicolumn{2}{|c|}{ COR } & \multicolumn{2}{|c|}{ NS } & \multicolumn{2}{|c|}{ MAE } & \multicolumn{2}{|c|}{ RMSE } & \multirow[t]{2}{*}{$\mathrm{RF}$} \\
\hline & & $V \max$ & Pmin & $\mathrm{P}$ & $\mathrm{V}$ & $\mathrm{P}$ & $\mathrm{V}$ & $\mathrm{P}$ & $\mathrm{V}$ & $\mathrm{P}$ & $\mathrm{V}$ & \\
\hline HWRF (27-9) & 4 & 7 & 8 & 7 & 8 & 3 & 8 & 1 & 2 & 2 & 3 & 5 \\
\hline HWRF (27-9-m) & 3 & 8 & 7 & 6 & 3 & 2 & 7 & 3 & 3 & 3 & 2 & 3 \\
\hline HWRF (18-6) & 1 & 5 & 2 & 4 & 5 & 4 & 2 & 4 & 1 & 4 & 5 & 2 \\
\hline HWRF (18-6-m) & 2 & 4 & 2 & 1 & 1 & 1 & 1 & 2 & 1 & 1 & 1 & 1 \\
\hline WRF - HWRF (27-9) & 5 & 6 & 6 & 8 & 7 & 6 & 6 & 5 & 5 & 8 & 8 & 8 \\
\hline WRF - HWRF(27-9-m) & 6 & 3 & 5 & 2 & 4 & 7 & 4 & 6 & 6 & 7 & 5 & 6 \\
\hline WRF - HWRF (18-6) & 7 & 2 & 4 & 3 & 6 & 8 & 5 & 7 & 7 & 5 & 6 & 7 \\
\hline WRF - HWRF (18-6-m) & 8 & 1 & 3 & 5 & 2 & 5 & 3 & 8 & 8 & 6 & 7 & 4 \\
\hline
\end{tabular}


Tabla 5 - Tiempo promedio de cómputo para cada configuración.

\begin{tabular}{lcccccccc}
\hline Modelo & HWRF & HWRF & HWRF & HWRF & WRF-HWRF & WRF-HWRF & WRF-HWRF & WRF-HWRF \\
\hline Dominio & $27-9$ & $27-9-\mathrm{m}$ & $18-6$ & $18-6-\mathrm{m}$ & $27-9$ & $27-9-\mathrm{m}$ & $18-6$ & $18-6-\mathrm{m}$ \\
Tiempo & $2 \mathrm{~h} \mathrm{50m}$ & $0 \mathrm{~h} 42 \mathrm{~m}$ & $3 \mathrm{~h} \mathrm{50m}$ & $3 \mathrm{~h} 58 \mathrm{~m}$ & $1 \mathrm{~h} 30 \mathrm{~m}$ & $1 \mathrm{~h} 2 \mathrm{~m}$ & $3 \mathrm{~h} 42 \mathrm{~m}$ & $2 \mathrm{~h} 48 \mathrm{~m}$ \\
\hline
\end{tabular}

nóstico operativo si no se cuenta con los medios de cómputo necesarios.

El tiempo de cómputo analizado en esta investigación se tomó a partir del inicio de la corrida del preprocesador de los datos geográficos geodrid.exe y concluyó con la ejecución de los algoritmos de postprocesamiento (búsqueda del centro de la tormenta en cada plazo de tiempo, evolución temporal de la velocidad máxima de los vientos y la presión mínima central, confección de mapas de trayectoria y campos meteorológicos de interés para el meteorólogo sinóptico).

En esta evaluación no se tuvo en cuenta el tiempo requerido para la descarga desde internet de los datos de inicialización de las configuraciones de los modelos, tiempo que en el proceso operativo de pronóstico es indispensable tomar en consideración. A partir de los resultados presentados en los epígrafes anteriores y teniendo en cuenta el tiempo de corrida, la configuración HWRF 27-9$\mathrm{m}$, es una alternativa viable para realizar un pronóstico operativo si existen limitantes en la capacidad de cómputo instalada.

\section{Conclusiones}

No se observaron diferencias significativas entre los resultados de las 8 configuraciones estudiadas en esta investigación. Esto se debe a que ambos modelos presentan similitudes en cuanto a la física utilizada para la representación del problema y a los métodos matemáticos empleados para la solución del sistema de ecuaciones que gobiernan el flujo atmosférico. Se observó un mejor comportamiento en las configuraciones asociadas al HWRF, debido a que este modelo está diseñado específicamente para el pronóstico de ciclones tropicales y la mayoría de las parametrizaciones que emplea están tropicalizadas.

Los errores en el pronóstico de la presión central oscilaron entre los 15 y $30 \mathrm{hPa}$, sin embargo, la curva de su valor medio decreció a medida que aumentó el plazo de tiempo mientras que los errores en el pronóstico de la velocidad máxima del viento oscilaron entre los 50 y $80 \mathrm{~km} / \mathrm{h}$. Igualmente, la curva de su valor medio tuvo un comportamiento atípico, similar al de la presión mínima. Al comparar las salidas del modelo con los registros de las boyas y estaciones meteorológicas de superficie se comprobó que el comportamiento de las variables estudiadas (velocidad del viento y presión atmosférica) presentó una tendencia similar a los valores registrados en las estaciones. La configuración que presentó los mejores resultados en las simulaciones realizadas fue HWRF 18-6-m aunque es la que más capacidad de cómputo requiere, razón por la cual es más recomendable emplear la configuración HWRF 27-9-m de forma operativa.

Los autores coinciden en que este es un estudio de caso único restringido al huracán Irma y que los resultados derivados pueden variar para otros huracanes, sin embargo, permite comprobar que el modelo HWRF es superior al WRF-HWRF. Además se verificó la necesidad de la incorporación de un esquema de relocalización de vórtice en la inicialización de los modelos para contar con una representación más detallada del huracán en concordancia con los reportes del NHC.

En próximas investigaciones se realizará una valoración del pronóstico numérico, que realiza cada configuración, del ambiente sinóptico en el que se desarrolla el huracán, se incorporarán nuevos casos de estudio, se realizará una evaluación detallada del pronóstico de precipitación asociada a los ciclones tropicales y se implementará un esquema de relocalización de vórtice para mejorar el campo de inicialización. encias

\section{Referencias}

ALIGO, E; FERRIER, B.; THOMPSON, G; CARLEY, J.R; ROGERS, E and DIMEGO, J. The New Ferrier-Aligo Microphysics in the NCEP 3-km NAM nest. In: Proceedings of the 97th AMS 492 Annual Meeting, Seattle, 2017.

ARAKAWA, A.; SCHUBERT, W.H. Interaction of a Cumulus cloud ensemble with the large-scale environment, Part I. Journal of the Atmospheric Sciences, v. 31, n. 3, p. 674$701,1974$.

BAO, J.-W.; GOPALAKRISHNAN, S.; MICHELSON, S.; MARKS, F. et al. Impact of physics representations in the HWRFX on simulated hurricane structure and pressurewind relationships. Monthly Weather Review, v. 140, n. 10 , p. 3278-3299, 2012.

BISWAS, M.K.; CARSON, L.; NEWMAN, K.; BERNARDET, L. et al. Community HWRF Users' Guide v. 3.9a, p. 160, 2017.

BORTNIKOV, S.A. Confección de un modelo multinivel de pronóstico basado en la experiencia del pronóstico operativo a corto plazo con las ecuaciones completas para una atmósfera de dos niveles. TrudiGMC, v. 16, p. 21-32, 1968.

CANGIALOSI, P.J. National Hurricane Center Forecast Verification Report. 2018 Hurricane Season. National Hurricane Center https://www.nhc.noaa.gov/verification/pdfs/ Verification_2018.pdf, 2019, Consultado: 14 de junio de 2019. 
CANGIALOSI, P.J.; LATTO, S.A.; BERG, R. National Hurricane Center Tropical Cyclone Report. Hurricane Irma (AL112017). National Hurricane Center https://www. nhc.noaa.gov/data/tcr/AL112017_Irma.pdf, 2018, Consultado: 14 de junio de 2019

CHEN, H.; GOPALAKRISHNAN, S.G. A study on the asymmetric rapid intensification of Hurricane Earl (2010) using the HWRF system. Journal of the Atmospheric Sciences, v. 72, n. 2, p. 531-550, 2018.

DEMARIA, M.; KNAFF, J.A.; KAPLAN, J. On the decay of tropical cyclone winds crossing narrow landmasses. Journal of Applied Meteorology and Climatology, v. 45, n. 3, p. 491-499, 2006.

DODLA, V.B.; DESAMSETTI, S.; YERRAMILLI, A. A comparison of HWRF, ARW and NMM models in Hurricane Katrina (2005) simulation. International Journal of Environmental Research and Public Health, v. 8, n. 6, p. 2447-2469, 2011.

FERRIER, B.S.; JIN, Y.; LIN, Y.; BLACK, T. et al. Implementation of a new grid-scale cloud and precipitation scheme in the NCEP ETA model. In: Proceedings of the 19th Conference on Weather Analysis and Forecasting/15th Conference on Numerical Weather Prediction. American Meteorological Society, 2002.

GALL, R.; FRANKLIN, J.; MARKS, F.; RAPPAPORT, E.E. et al. The Hurricane Forecast Improvement Project. Bulletin of the American Meteorological Society, v. 94, n. 3, p. 329-343, 2013,

GOPALAKRISHNAN, S.G.; GOLDENBERG, S.; QUIRINO, T.; ZHANG, X. et al. Toward improving high-resolution numerical hurricane forecasting: Influence of model horizontal grid resolution, initialization, and physics. Weather and Forecasting, v. 27, n. 3, p. 647-666, 2012.

GOPALAKRISHNAN, S.G.; ZHANG, J.A.; ZHANG, X.; BAO, J.-W. et al. A study of the impacts of vertical diffusion on the structure and intensity of the tropical cyclones using the high-resolution HWRF system. Journal of the Atmospheric Sciences, v. 70, n. 2, p. 524-541, 2013.

GOPALAKRISHNAN, S.G.; MARKS, F.; ZHANG, X.; BAO, J.-W. et al. The experimental HWRF system: A study on the influence of horizontal resolution on the structure and intensity changes in tropical cyclones using an idealized framework. Monthly Weather Review, v. 139, n. 6, p. 1762-1784, 2011.

HAN, J.; PAN, H.-L. Revision of Convection and Vertical Diffusion Schemes in the NCEP Global Forecast System. Weather and Forecasting, v. 26, n. 4, p. 520-533, 2011.

HAN, J.; WANG, W.; KWON, Y.C; HONG, S. et al. Updates in the NCEP GFS Cumulus Convection Schemes with Scale and Aerosol Awareness. Weather and Forecasting, v. 32, n. 5, p. 2005-2017, 2017.

HOLT, C.; BERNARDET, L.; BROWN, T.; YABLONSKY, R.. Community HWRF Users' Guide v3.6a. NOAA-ESRLGSD, Developmental Testbed Center and CIRES-CU, 2014.

HONG, S.-Y.; PAN, H.-L. Nonlocal boundary layer vertical diffusion in a medium-range forecast model. Monthly Weather Review, v. 124, p. 2322-2339, 1996.

JANJIC, Z.; BLACK, T.; PYLE, M.; FERRIER, B. et al. NMM Version 3 Modelling System User's Guide. Develop- mental Testbed Center / National Centers for Enviromental Prediction, 2014.

KO, M.; MARKS, F.; ALAKA, G.J.; GOPALAKRISHNAN, S.G. Precipitation evaluation of the real-time basin-scale HWRF in 2017. In: 33rd Conference on Hurricanes and Tropical Meteorology. American Meteorological Society, 2017.

LIGHTHILL, J. Fluid mechanics of tropical cyclones. Theoretical and Computational Fluid Dynamics, v. 10, n. 1, p. 3-21, 1998 .

MITRANI, A.I.; BORRAJERO, M.I.; MARTÍNEZ, C.D.; ALONSO, D.Y. et al. Predicción del oleaje en presencia de los huracanes "Iván" y "Paloma", mediante el empleo de la combinación de los modelos numéricos MM5V3-WW3. Revista Cubana de Meteorología, v. 17, n. 2, p. 39-48, 2011.

MITRANI, A.I.; GONZÁLEZ, P.C. Experiencias con el uso de MM5V3 al paso de los huracanes "Isidore" y "Lili" por territorio cubano. Revista Brasileira de Meteorología, v. 20, n. 2, p. 227-242, 2005

MITRANI, A.I.; PÉREZ, B.; CABRALES, I.J.; PÉREZ, P.Y. et al. Coastal flood forecast in Cuba, due to hurricanes, using a combination of numerical models. Revista Cubana de Meteorología, v. 25, n. 2, p. 121-138, 2019.

MITRANI, A.I.; PÉREZ, B.A.; VICHOT, L.A.; ALONSO, D.Y. et al. Numerical forecast of weather and wind waves, using WRF and WW3, on the Cuban territory and surrounding waters, and comparison with MM5+WW3. Ciencias de la Tierra y el Espacio, v. 18, n. 2, p. 86-100, 2017.

MITRANI, A.I.; ÁLVAREZ, E.L.; BORRAJERO, M.I. Aplicación optimizada del MM5V3 sobre el territorio cubano mediante el uso de una computadora personal. Revista Cubana de Meteorología, v. 10, n. 1, p. 84-94, 2002.

MITRANI, I.A.; PÉREZ, J. Aplicación de un modelo de alta resolución en la predicción del tiempo sobre territorio cubano, con posibilidad de pronosticar el movimiento de los ciclones tropicales. Boletín SOMETCUBA, v. 3, n. 2, 1999.

MITRANI, I.A.; MARTÍNEZ, C.D.; BORRAJERO, M.I.; HICKS, E. et al. Numerical weather prediction and tropical cyclone track forecasting in the Caribbean using MM5. Case studies of hurricanes Dean (2007), Omar (2008) and Paloma (2008). 29th Conference on Hurricanes and Tropical Meteorology, Available en https://ams.confex.com/ ams/29Hurricanes/techprogram/paper169389.htm, 2010, Consultado: 28 de mayo de 2019

NASH, J.E.; SUTCLIFFE, J.V. River flow forecasting through conceptual models. Part I - A discussion of principles. Journal of Hydrology, v. 10, n. 3, p. 282-290, 1970.

NIKKI C.P.; RONALD M.E. The role of model and initial condition error in numerical weather forecasting investigated with an observing system simulation experiment. Tellus A: Dynamic Meteorology and Oceanography, v. 65, n. 1, p. $21740,2013$.

PÉREZ-BELLO, A.; MITRANI, A.I.; DÍAZ, R.O.; WETTRE, C. et al. A numerical prediction system combining ocean, waves and atmosphere models in the Inter-American Seas and Cuba. Revista Cubana de Meteorología, v. 25, n. 1, p. 109-120, 2019. 
SIERRA, L.M.; FERRER, H.A.L.; VALDÉS, R.; MAYOR, G.Y. et al. Sistema automático de predicción a mesoescala de cuatro ciclos diarios. In: Informe de Resultado. La Habana: Instituto de Meteorología, 2015.

SINGH, K.S.; MANDAL, M. Impact of initial and boundary conditions on mesoscale simulation of Bay of Bengal cyclones using WRF-ARW model. In: Ray, K.; Mohapatra, M.; Bandyopadhyay, B.; Rathore, L. (eds) High-Impact Weather Events over the SAARC Region. Cham: Springer, 2015.

SMITH, R.K.; THOMSEN, G.L. Dependence of tropical-cyclone intensification on the boundary-layer representation in a numerical model. Quarterly Journal of the Royal Meteorological Society, v. 136, n. 132, p. 1671-1685, 2010.

TRAN, D.; CHIAO, S. Evaluating HWRF modeling of landfallling tropical cyclones over Florida. In: Especial Symposium on The Next Level of Predictions in Tropical Meteorology, Techniques, Usage, Support and Impacts, American Meteorological Society, 2013.
ZHANG, J.A.; MARKSs, F. Effects of horizontal diffusion on tropical cyclone intensity change and structure in idealized three-dimensional numerical simulations. Monthly Weather Review, v. 143, n. 10, p. 3981-3995, 2015.

\section{Recursos de Internet}

NOAA / National Data Buoy Center (NDBC), http://www.ndbc. noaa.gov/rmd.shtml.

HURDAT2, NOAA / National Hurricane Center (NHC), https:// www.nhc.noaa.gov/data/\#hurdat.

NOAA / National Climatic Data Center (NCDC), https:// nomads.ncdc.noaa.gov/data/gfs4/.

License information: This is an open-access article distributed under the terms of the Creative Commons Attribution License (type CC-BY), which permits unrestricted use, distribution and reproduction in any medium, provided the original article is properly cited. 